\title{
CO-ORDINATED OPTICAL AND RADIO \\ OBSERVATIONS OF SYMBIOTIC STARS
}

\author{
R. J. Ivison and M. F. Bode \\ School of Physics \& Astronomy, Lancashire Polytechnic, Corporation St., Preston, PR1 2TQ, U.K. \\ J. Meaburn \\ Department of Astronomy, University of Manchester, Manchester, M13 9PL, U. K. \\ R. J. Davis, R. F. Nelson and R. E. Spencer \\ Nuffield Radio Astronomy Laboratories, Jodrell Bank, Macclesfield, Cheshire, SK11 9DL, U.K.
}

\begin{abstract}
We present preliminary results from absolutely flux-calibrated optical spectroscopy, together with $5 \mathrm{GHz}$ radio flux measures of 17 symbiotic stars. The data were obtained quasi-simultaneously using the Manchester Echelle Spectrograph on the Isaac Newton Telescope, La Palma between 1988 September 20 and 24, and the Broad Band Interferometer at Jodrell Bank during 1988 October. This represents the largest sample of these stars observed in this way to date. Distances are calculated using visual extinctions and are compared with other quoted values. In general, the agreement is surprisingly good. Plots of individual line luminosities vs. radio luminosity indicate that the D-type (dusty) symbiotics (both also proto-planetary nebulae) have far higher radio luminosities than S-types (stellar) for comparable recombination and forbidden line luminosities. This may however be due to underestimation of optical line luminosities for the D-types as the circumstellar extinction contribution is uncertain. The single $\mathrm{D}$ '-type (cool dust, yellow secondary) lies at the low luminosity end of the S-type region. 'Very slow novae' in our sample lie in both groups.
\end{abstract}

\section{Introduction}

Symbiotic stars are thought to be interacting binary systems, but unlike classical novae the mass-donating secondary is a red giant which in most cases is probably not filling its Roche lobe. Mass transfer on to the hot component in this case would be via a wind.

Several stars (eg. V1016 Cyg, HM Sge, AG Peg, PU Vul and V1329 Cyg) have shown large amplitude outbursts, developing over many years, and have often been dubbed 'very slow novae' (Vogt 1989). Indeed their outbursts may well arise from thermonuclear events on white dwarf surfaces. However, the true nature of the hot component remains unclear in the vast majority of cases, and hence the cause of outbursts is much more speculative than in classical novae.

We are currently conducting a complete high resolution optical spectroscopic survey of symbiotics in conjunction with groups at Calgary, Keele, Manchester and Münster. From this work we hope to gain insight into the nature of the hot components, accretion processes, outburst mechanisms and the environments of symbiotic stars. As part of this survey, we have routinely obtained absolutely flux-calibrated low resolution spectra (see Fig. 1). This was done on our 1988 September observing run on La Palma, but we were fortunate enough to be able to arrange $5 \mathrm{GHz}$ observations from Jodrell Bank within a few weeks of our optical work. Although comparison of radio

\begin{tabular}{|c|c|c|c|c|c|c|c|c|}
\hline Object & $\begin{array}{c}5 \text { GHz } \\
\text { Radio Flux } \\
(\mathrm{m} J \mathbf{J})\end{array}$ & $\begin{array}{l}\text { Line FI } \\
\text { HeII } \\
4686\end{array}$ & $\frac{\operatorname{uxps}(x}{\mathrm{H} \beta}$ & $\frac{10^{-10} \mathrm{~W}}{\text { TOIII) }}$ & $\frac{\left(\mathrm{cm}^{-3}\right)}{\frac{\mathrm{H} \alpha}{26563}}$ & $\mathbf{E}_{\boldsymbol{B}-\boldsymbol{v}}$ & $\begin{array}{c}\text { Extinction } \\
\text { Dintance } \\
\text { (pc) }\end{array}$ & $\begin{array}{c}\text { Previous } \\
\text { Distance } \\
\text { (pc) }\end{array}$ \\
\hline EG And & $<0.81$ & $<1.8$ & $<1.3$ & $<2.8$ & $<2.8$ & 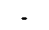 & - & 630 (I) \\
\hline 2 And & $1.35 \pm 0.30$ & 36 & 53 & $<1$ & 133 & 0.57 & 900 & $\begin{array}{l}2190(1) \\
1230(2)\end{array}$ \\
\hline AS 360 & $<1.45$ & $<0.2$ & $<0.3$ & $<0.3$ & $5.2 ;$ & - & - & $10^{4}(3)$ \\
\hline UV Aur & $<28.5 \#$ & $<8$ & 87 & $<\theta$ & 252 & 0.80 & 1800 & - \\
\hline BF Cyg & $2.06 \pm 0.43$ & $<3$ & 47 & 13 & 134 & 0.31 & 1000 & - \\
\hline $\mathrm{CH} \mathbf{C y y}_{\mathbf{B}}$ & $1.81 \pm 0.29$ & $<3$ & 3: & $<2$ & $<8$ & - & - & - \\
\hline $\mathrm{Cl} \mathrm{Cyz}$ & $<0.51$ & $<3$ & 52 & $<5$ & 150 & 0.47 & 1200 & $2100(1)$ \\
\hline V407 Cyg & $<2.14$ & $<0.1$ & $<0.1$ & $<0.1$ & $<0.2$ & - & - & - \\
\hline $\mathrm{V}_{1016} \mathrm{C}_{\mathrm{Y}}$ & $43.0 \pm 1.1$ & 107 & 270 & 305 & 509 & 0.50 & 1000 & $1000(4)$ \\
\hline $\mathrm{v}_{1329} \mathrm{Cyg}_{\mathrm{g}}$ & $1.81 \pm 0.43$ & 48 & 04 & 8 & 164 & 0.92 & 2300 & 1675 (4) \\
\hline He2 - 46T & $<0.64$ & $<0.2$ & $<0.1$ & $<0.1$ & $<0.2$ & - & - & - \\
\hline He2 - 468 & $<0.94$ & $<0.3$ & $<0.5$ & $<0.1$ & 1.7: & - & - & - \\
\hline AG Peg & $8.61 \pm 0.48$ & 38 & 79 & $<0$ & 230 & 0.16 & 800 & $800(3)$ \\
\hline AX Per & $<1.11$ & $<20$ & 132 & $<21$ & 394 & 0.89 & 2200 & $2820(1)$ \\
\hline v741 Per & $<0.94$ & $<0.4$ & 4.5 & 7.0 & 12.8 & 0.37 & 800 & - \\
\hline HM Sge & $34.6 \pm 1.4$ & 20 & 11 & 145 & 123 & 0.32 & 800 & $1000(3)$ \\
\hline Pu Vul & $<0.53$ & $<14$ & 480 & $<36$ & 1401 & 0.09 & 2300 & - \\
\hline
\end{tabular}

Table 1. Radio and dereddened optical line fluxes. Colour excesses calculated from $(\mathrm{H} \alpha / \mathrm{H} \beta)$ using case $\mathrm{B}$ recombination theory. Distances calculated using the extinction maps of Lucke (1978). Errors are typically $10 \%$ on line fluxes, \pm 0.13 on extinctions, and $50 \%$ on distances.

Notes. \# Probably confused. : Possible large error.

Refs. (1) Kenyon \& Gallagher, 1983. (2) Altamore et al, 1981. (3) Allen, 1980. (4) Blair et al, 1983. 
and optical observations of symbiotics have been made previously (see e.g. Seaquist et al 1984; Seaquist 1988) these have generally been separated by long timescales. As pointed out by Slovak and Code (1988), because most symbiotics are variable, the multi-frequency data should ideally be obtained as simultaneously as possible, which was our aim here. In addition, previous published optical data used in this way have generally not been absolutely flux-calibrated. This paper gives details of our observations and preliminary work on their analysis.

\section{Discussion}

Figure 2 shows that the stars we have observed fall into 2 distinct groups, the S-types and the $D$-types. Indeed it has been known for several years that the $\mathrm{D}$-types are generally brighter in the radio (Seaquist et al 1984; Seaquist 1988). Here we find they have much higher radio luminosities than the S-types for equivalent $\mathrm{H} \alpha$ luminosities. This pattern is repeated in plots of radio luminosity against [O III], $\mathrm{H} \beta$ and $\mathrm{He}$ II line luminosities. We note too that there are examples of very slow novae in both groups. Intriguingly, the single D'type (V741 Per) lies at the low luminosity end of the S-types.

As both the thermal radio emission and the Balmer lines arise from the same volume of gas, underestimation of radio luminosity could be due to greater optical depth in the radio for the Sthan the D-types. Alternatively, we may have underestimated the extinction through the D-types' circumstellar dust shells. This would arise if the extinction law of circumstellar dust were not the same as that in the interstellar medium. Work is continuing to explore these possibilities further.

We are currently attempting to obtain better reddenning estimates than the $\mathrm{H} \alpha / \mathrm{H} \beta$ ratio, which is prone to error if $\mathrm{H} \alpha$ is optically thick. Physical parameters of the ionized gas will then be determined. Further simultaneous multi-frequency observations are now scheduled.

\section{References}

Allen, D. A., 1980. Mon. Not. R. astron. Soc., 192, 521.

Altamore, A. et al, 1981. Astrophys. J., 245, 630.

Blair, W. P. et al, 1983. Astrophys. J. Suppl., 53, 573.

Kenyon, S. J. \& Gallagher, J.S., 1983. Astron. J., 88, 666.

Lucke, P. B., 1978. Astron. Astrophys., 64, 364.

Seaquist, E. R. et al, 1984. Astrophys. J., 284, 202.

Seaquist, E. R., 1988. in 'The Symbiotic Phenomenon', eds Mikolajewska et al, Kluwer, p 69.

Slovak, M.H. \& Code, A.D., 1988. in 'The Symbiotic Phenomenon', eds Mikolajewska et al, Kluwer, p 43.

Vogt, N., 1989. in 'Classical Novae', eds Bode \& Evans, Wiley, p 239.
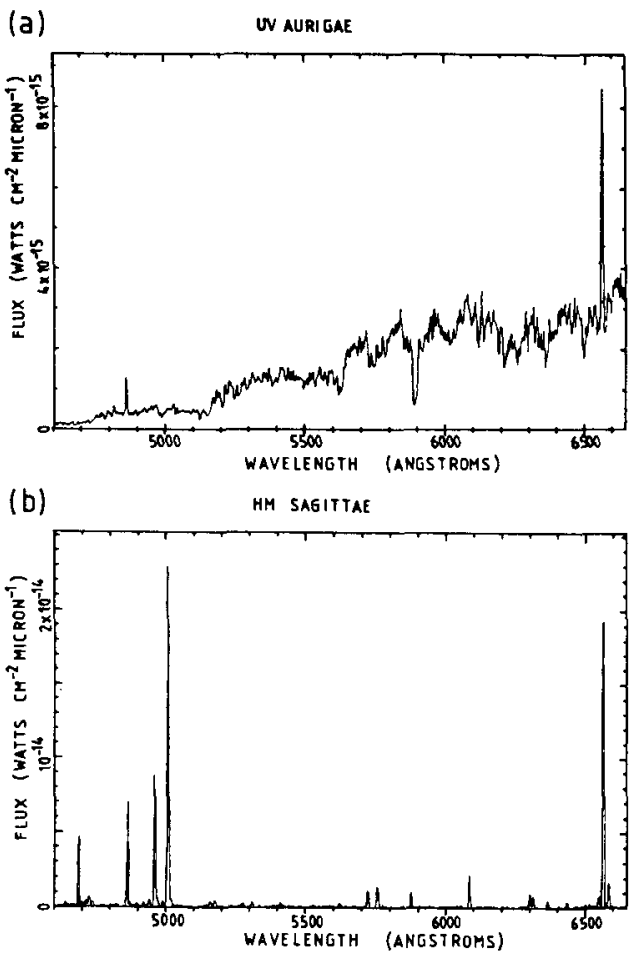

Figure 1. Optical spectra of selected stars. (a) UV Aurigae (b) HM Sagittae.

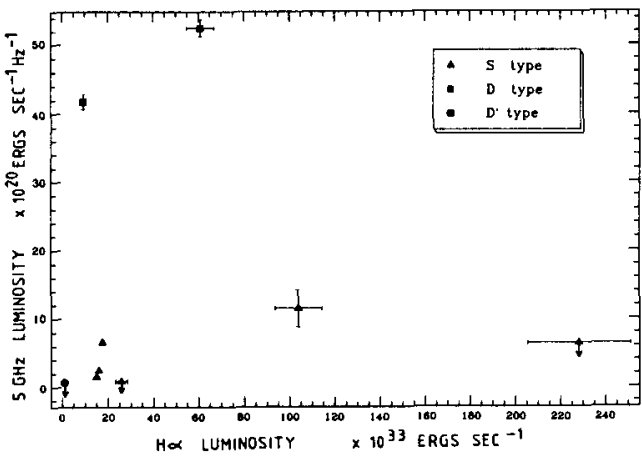

Figure 2. $\mathrm{H} \alpha$ optical line luminosities vs. $5 \mathrm{GHz}$ radio luminosities based on our extinction distances. 